\title{
The State of the Art in Feature Extraction Methods for Electroencephalogram Epileptic Classification
}

\author{
Mokhtar Mohammadi', Hoger Mahmud² \\ ${ }^{1}$ Department of Information Technology, College of Science and Technology, University of Human Development, \\ Sulaymaniyah, Kurdistan Region, Iraq, ${ }^{2}$ Department of Computer Science, College of Science and Technology, University \\ of Human Development, Sulaymaniyah, Kurdistan Region, Iraq
}

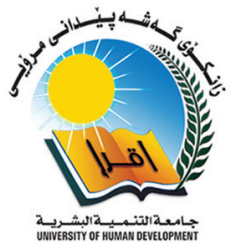

\section{A B S T R A C T}

Epilepsy is a neurological disease that is common around the world, and there are many types (e.g., Focal aware seizures and atonic seizure) that are caused by epileptic seizures. An epileptic seizure is a transient of symptoms because of abnormal excessive or synchronous neural activity in the brain. Electroencephalogram (EEG) is a common way to record brain activity brain activities generated by nerve cells in the cerebral cortex. Automatic epileptic seizure detection or prediction system can classify normal from abnormal EEG signal. Selection of discriminant features is a matter of the performance of an automatic system. In this paper, we review several features extracted from the time, frequency, and time-frequency domains proposed by different researches for the purpose of epileptic seizure detection, also analyze, and compare the performance of the proposed features.

Index Terms: Classification, Electroencephalogram, Epileptic Seizure Detection, Feature Extraction, Time-frequency Analysis

\section{INTRODUCTION}

An epileptic seizure is one of the most common neurological disorders caused by brain activity impulses that escape their boundaries and affect other areas of the brain through creating a storm of electrical activities [1-3]. An epileptic seizure is the result of excessive neuronal spontaneous and synchronized discharge in the group of the brain cells. To detect symptoms of epileptic seizure in a patient, electroencephalography (Electroencephalogram [EEG]) is used commonly [3,4]. EEG measures the electrical activity of the brain and generates a dynamic visual image of the

\begin{tabular}{|l|l|}
\hline \multicolumn{2}{|c|}{ Access this article online } \\
\hline DOI:10.21928/uhdjst.v3n2y2019.pp16-23 & $\begin{array}{l}\text { E-ISSN: 2521-4217 } \\
\text { P-ISSN: 2521-4209 }\end{array}$ \\
\hline
\end{tabular}

Copyright (C) 2019 Mohammadi and Mahmud. This is an open access article distributed under the Creative Commons Attribution NonCommercial No Derivatives License 4.0 (CC BY-NC-ND 4.0) brain activities that can be scanned for abnormalities that may indicate whether the patient is suffering from epileptic seizure or not.

Visually inspection of EEG recordings is time consuming and requires specialists such as neurophysiologists to analyze the recordings and diagnose the case. To facilitate the detection of epileptic seizure signs with high accuracy and reduce the time taken to make diagnostics, it is essential that an automated computer-based system to be utilized $[5,6]$.

To use EEG recordings and make a diagnostic, the following steps will have to be taken:

1. Preprocessing

2. Analyzing EEG recordings using the time, frequency, and joint time-frequency domain

3. Identify patterns that indicate seizure activities (feature extraction)

4. Classify identified patterns to make correct diagnostics.

Corresponding author's e-mail: Hoger Mahmud, Department of Computer Science, College of Science and Technology, University of Human Development, Sulaymaniyah, Kurdistan Region, Iraq. E-mail: hoger.mahmud@uhd.edu.iq 
Feature extraction as an important step in automatic epileptic seizure detection system has attracted lots of attentions. Some of the researchers such as [7] used time domain to extracts features from EEG data; [8] used frequency domain for feature extraction; and the combination of time and frequency domains are also used by researchers in Boubchir et al. [6], Boubchir et al. [8], and Boubchir et al. [9]. Reference Mohammadi et al. [10] proposed time-frequency features to detect epileptic seizure using EEG recordings. Depend on the selected domain, different features have been proposed for epileptic seizure detection.

Amplitude modulation (AM)-frequency modulation (FM) signals can be used to model real-life signals and the model is normally recognized by attributes such as instantaneous phase, instantaneous frequency (IF), and instantaneous amplitude (IA); these attributes, when extracted, can yield good classification outcomes $[11,12]$. To extract IF or IA, methods such as TED and empirical mode decomposition can be used [13], as for differentiating signals with high signal energy, measures such as Renyi entropy and time-frequency flatness are good choices of use. The two measures are types of time-frequency entropy measures that are good indicators of seizure activity when EEG signals are considered [12]. In identifying seizure activities, the shape and direction of energy distribution in time-frequency signals are important; these features can be extracted using directional or wavelet decomposition filters and the features can be captured in a number of images. The images can later be used to obtain statistical features [14]. There are other methods that can be used for the same extraction purpose, such as dimensionality reduction methods proposed by Sameh and Lachiri [14].

In this paper, we analyze and compare different features that have been proposed by various researchers as proofs for seizure activities and classifications. We found out that the features extracted from the joint time-frequency domain provide more advantageous than those extracted from either time or frequency domain. The structure of the paper is as follows:

In the following, we discuss the framework for EEG classification. In the next section, we review the feature extraction methods, and then, we discuss the performance of several EEG features. Finally, we conclude the paper.

\section{EEG SEIZURE DETECTION AND CLASSIFICATION FRAMEWORK}

In this section, we explain the steps of seizure detection process using EEG that includes five steps as below:

\subsection{The Preprocessing Stage}

In this stage, we remove noise and excess features from EEG recordings, using techniques such as band-pass filtering and Bayesian denoising [15]. This stage will prepare the data for processing and facilitate correct seizure-related signal detection and clears away the unwanted artifacts that may distort the real result.

\subsection{EEG Signal Representation}

To ensure that the best EEG signal representation is used in seizure diagnostics, it is necessary to decide in what representation domain signals are analyzed. The typical representation domains used by researchers are time, frequency, and joint time-frequency domains. (Fig. 1a) shows a normal EEG signal in the time domain. (Fig. 1b) shows the time-frequency representation of the same signal in the joint time-frequency domain. Fig. 2a shows an abnormal EEG signal and its time-frequency representation. From (Fig. 2b), we can observe a train of spikes in the time-frequency representation of an epileptic EEG signal.

\subsection{Feature Extraction}

At this stage features and patterns, indicating seizure activities are extracted from the preprocessed EEG data. The common features that are proposed by researchers are classified into four categories. The first is known as (amplitude-based) extracted from EEG signal in time domain, the second is (spectrum-based) extracted in the frequency domain, the third is (IF) extracted in the time-frequency domain [9], and the fourth is (image descriptor-based) extracted in timefrequency domain $[6,8]$.

\subsection{Feature Selection}

As a result of the previous steps, a number of features are extracted; however, not all features are decisive in seizure diagnostics as there will be redundant or irrelevant features in the extraction. In this step, it is required to filter the most irrelevant features and eliminate the unwanted ones to ensure the quality and correctness of the final seizure diagnostics and classification.

\subsection{Classification}

The features extracted and filtered in the previous step are now ready to be used for the final diagnostics and classification. In this step, several classifiers such as an artificial neural network and support vector machine are used for the classification. A cross-validation method is used to evaluate the performance of the classifier. The leave-one-out technique can be used for validation, which gives an almost unbiased approximation of the true generalization error. 


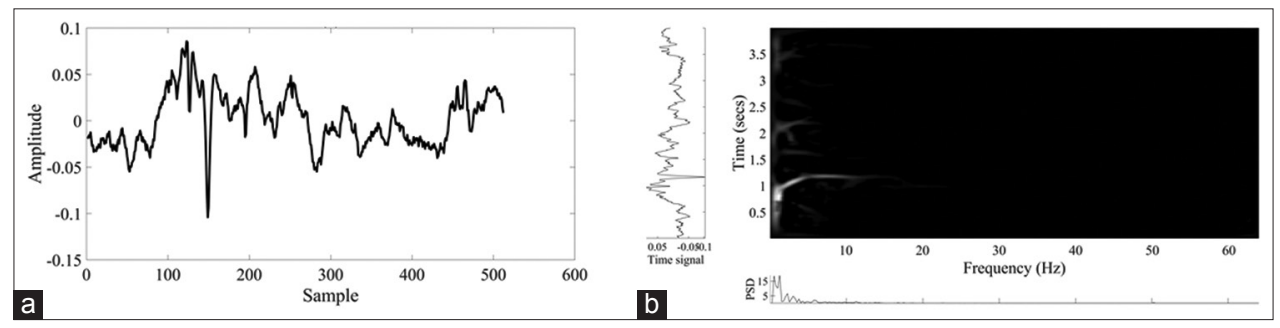

Fig. 1. (a) An electroencephalogram signal with normal activity and (b) time-frequency representation of the signal.
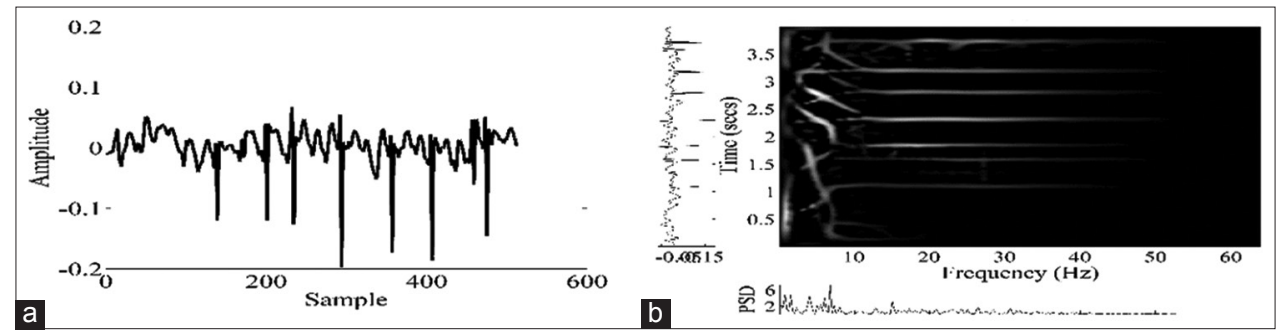

Fig. 2. (a) An electroencephalogram signal with epileptic seizure activity and (b) time-frequency representation of the signal.

The performance of the classifier can be assessed based on specificity, sensitivity, and total accuracy.

Specificity $=\frac{\text { True Negative }}{\text { Total Number of True Negatives }}$

Sensitivity $=\frac{\text { True Positive }}{\text { Total Number of True Positives }}$

Total Accuracy $=\frac{\text { True Positive }+ \text { True Negative }}{\text { Total Number of Examples }}$

Fig. 3 shows a flowchart indicating the input, computational steps, and output of a classification system.

\section{EEG FEATURE EXTRACTION REVIEW}

Features extracted form time, frequency, or $\mathrm{t}-\mathrm{f}$ representations are the most widely proposed features for EEG seizure detection; in this section, we present a brief literature review of the features.

A. Time-domain features: To extract seizure indicator features, the median absolute deviation or root mean square or inter-quartile range of the amplitude of EEG signals are scanned in the time domain [16-18]. Below we present some other features that are suggested by researchers for extraction such as statistical moments $[16,17,19]$.
1. Features based on statistical moments:

- First moment and second central moment of EEG signal $[16,19]$

Mean:

$F_{1}^{(t)}=\mu=\frac{1}{N} \sum_{n=1}^{N}|z[n]|$

Variance:

$F_{2}^{(t)}=\sigma^{2}=\frac{1}{N} \sum_{n=1}^{N}(\mu-|z[n]|)^{2}$

- Normalized moments: Third and fourth central moments of EEG signal [16,19]

Skewness:

$$
F_{3}^{(t)}=\frac{1}{N^{\sigma 3}} \sum_{n=1}^{N}(|z[n]|-\mu)^{3}
$$

Kurtosis:

$F_{4}{ }^{(t)}=\frac{1}{N^{\sigma 4}} \sum_{n=1}^{N}(|₹[n]|-\mu)^{4}$

- Coefficient of variation of the EEG signal [19]

$F_{5}^{(t)}=\frac{\sigma}{\mu}=\sqrt{\frac{F_{2}^{(t)}}{F_{1}^{(t)}}}$ 


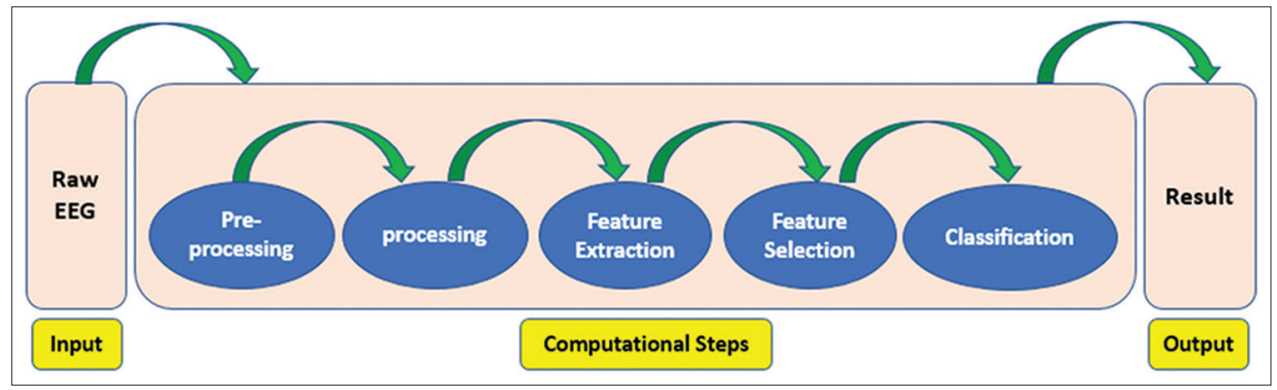

Fig. 3. The flowchart of electroencephalogram classification system.

2. Features based on amplitude:

- Median absolute deviation of EEG amplitude [16]

$F_{6}^{(t)}=\frac{1}{N} \sum_{n=1}^{N}(|z[n]-\mu|)$

- $\quad$ Root mean square amplitude [17]

$F_{7}^{(t)}=\sqrt{\frac{\sum_{n=1}^{N} z[n]^{2}}{N}}$

- Interquartile range [18]

$F_{8}^{(t)}=q\left[\frac{3(N+1)}{4}\right]-z\left[\frac{(N+1)}{4}\right]$

3. Features based on entropy:

- Shannon entropy $[16,17,20]$

$F_{9}^{(t)}=-\sum_{n=1}^{N} z[n] \log _{2}(z[n])$

B. Frequency domain features: The frequency representation of EEG signal is scanned in this domain to identify seizure indicator features based on spectral information (e.g., power spectrum, spectral Roll-Off) $[16,17,19]$. Below we summarize some features extracted in frequency-domain.

1. Features based on power spectrum:

- Maximum power of the frequency bands [17,19]

$F_{1}^{(f)}=\sum_{k=1}^{\delta}|Z[k]|^{2}$

$F_{2}^{(f)}=\sum_{k=\delta+1}^{M}|Z[k]|^{2}$

- M corresponds to the maximum frequency

2. Features based on spectral information:

- Spectral centroid: Average signal frequency weighted by the magnitude of spectral centroid [16]
$F_{3}^{(f)}=\frac{\sum_{k=1}^{M} k|Z[k]|}{\sum_{k=1}^{M}|Z[k]|}$

- Spectral flux: Difference between normalized spectra magnitudes [16]

$F_{4}^{(f)}=\sum_{k=1}^{M}\left(Z^{(l)}[k]-Z^{l-1}[k]\right)^{2}$

Where $z^{(i)}$ and $z^{(i-1)}$ are normalized magnitude of the Fourier transform at $i$ and $i-1$ frames

- Spectral flatness: Indicates whether the distribution is smooth or spiky [16]

$F_{5}^{(f)}=\left(\Pi_{k=1}^{M} Z[k]\right)^{\frac{1}{M}}\left(\sum_{k=1}^{M} Z[k]^{-1}\right.$

- Spectral Roll-Off: Spectral concentration below threshold $\lambda[16]$

$F_{6}^{(f)}=\lambda \sum_{k=1}^{M}|Z[k]|$

3. Feature based on entropy:

- Spectral entropy: Measures the regularity of the power spectrum of EEG signal [17]

$F_{7}^{(f)}=\frac{1}{\log (M)} \sum_{k=1}^{M} P(Z[k]) \log P(Z[k])$

C. Time-frequency domain features: The joint timefrequency domain representation is more informative for the analysis of real-life signals. This indicates that if the additional information provided by the time-frequency representation is properly extracted in the form of timefrequency features, then better classification accuracy can be achieved. In this domain, the EEG signals are scanned for features that indicate seizure activities based 
on information extracted from both time and frequency domain.

Several techniques are available for the extraction, and the most widely used ones are:

1. IF features: Many real-life signals can be modeled as AM-FM signals. Such signals are completely characterized by the parameters of the AM-FM model that is the instantaneous phase, I), IA, and a total number of components. For such signals, parameters extracted from the IF or IA can lead to good classification results [15-18]. The IF or IA related parameters can be extracted either from time-frequency distributions (TFDs) or empirical mode decomposition based methods $[9,18,19]$.

2. Using image descriptors and image processing techniques such as shape and texture descriptor and local binary pattern (LBP) descriptor to scan time-frequency image representation of EEG signals for seizure indicator features $[6,8]$.

3. Entropy features: Time-frequency entropy measures such as Renyi entropy, time-frequency flatness can be used for discriminating signals having a high concentration of signal energy from signals having energy spread in the time-frequency domain [9,21], for example, in the case of EEG signals, seizure activity is sparse in the time-frequency domain, while the background is not.

4. Texture features: Texture time-frequency features are related to the direction and shape of energy distribution. These features can be obtained by, convolving a TFD with a set of convolution masks such as wavelet decomposition filters or directional filters to obtain a number of filtered images.

5. Other approaches include dimensionality reduction methods for directly extracting features from given TFDs, time-frequency matched filtering and statistical features $[10,20,22,23]$.

In the following, we describe the relevant t-f EEG features that we have identified. These features are based on IF [5], entropy [5], flux, flatness, and energy information of EEG signal (e.g., sub-bands energies and energy localization) [9,5]; which are computed from $\varrho$. Time-frequency image relatedfeatures: Other time-frequency features have been recently proposed based on image descriptors capable to describe visually the seizure activity pattern observed in the TFD of EEG signal, $\varrho$, considered and processed as an image using image processing techniques. The proposed time-frequency image features include shape and texture descriptors [6], Haralick descriptor [24], and LBP descriptor [8].
1. Features based on energy:

- Sub-bands energies [9]:

$$
\begin{aligned}
& F_{1}^{(t)}=\sum_{n=1}^{N} \sum_{k=1}^{M_{\delta}} \rho[n, k] \\
& F_{2}^{(f f)}=\sum_{n=1}^{N} \sum_{k=M_{\delta}+1}^{M_{\delta}} \rho[n, k]
\end{aligned}
$$

Where $M_{\delta}=M / f_{\delta}$ and $M$ corresponds to a maximum frequency component in the signal $\left(f_{\delta} / 2\right)$

- Energy localization [5]:

$F_{3}^{(f)}=\frac{\left(\Pi_{n=1}^{N} \Pi_{k=1}^{M} \rho[n, k]\right)^{\frac{1}{N M}}}{\sum_{k=1}^{M} \sum_{n=1}^{N} \rho[n, k]}$

2. Features based on IF:

- Mean and deviation of IF of EEG signal

$F_{4}^{(f)}=\frac{1}{N} \sum_{n=1}^{N} f_{i}[n]$

$F_{5}^{(f)}=\max f_{i}[n]-\min f_{i}[n]$

Where $f_{i}[n]=\frac{f_{\delta}}{2 M} \frac{\sum_{k=1}^{M} k \rho[n, k]}{\sum_{k=1}^{M} \rho[n, k]}$

3. Feature-based on entropy:

- Re'nyi entropy of order $\alpha[10]$

$F_{6}^{(f f)}=\frac{1}{1-\alpha} \log _{2}\left(\sum_{n=1}^{N} \sum_{k=1}^{M} \rho^{\alpha}[n, k]\right)$

- Normalized Renyi entropy

TFRE $=-\frac{1}{2} \log _{2} \sum_{n=1}^{N} \sum_{k=1}^{N}\left(\frac{\rho[n, k]}{\sum_{n} \sum_{k} \rho[n, k]}\right)^{3}$

- Time-frequency flatness

$T F_{\text {Flatness }}=N^{2} \frac{\prod_{n=1}^{N} \prod_{k=1}^{N} \rho[n, k]}{\sum_{n=1}^{N} \sum_{k=1}^{N} \rho[n, k]}$

UHD Journal of Science and Technology | Jul 2019 | Vol 3 | Issue 2 
4. Time-frequency flux:

$$
\mathrm{TF}_{\mathrm{FLUX}}=\sum_{l=1}^{N} \sum_{m=1}^{N} \rho[n+l, k+m]-\rho[n, k]
$$

\section{ANALYSIS AND DISCUSSION}

We have compared and analysis the EEG seizure detection and classification methods that use the EEG features described in Section III. We have considered here only the state-ofthe-art methods that have been assessed on Bonn University EEG database [23] and Freiburg EEG dataset [25] which are public free database widely used. This database includes five EEG sets referred to as sets A-E where each set contains 100 artifact-free EEG signals of $23.6 \mathrm{~s}$ duration acquired from normal subjects and patients with epileptic seizures. All the EEG signals in the database have been recorded at fs $=173.6 \mathrm{~Hz}$ sampling rate thus resulting in 4096 samples $(=23.6 \times \mathrm{fs})$ and have the spectral bandwidth varying from 0.5 to $85 \mathrm{~Hz}$ (see [23] for more detail). For all the methods considered in this review, which used the bone database, the desired classification is given in two different classes of EEG signals: Normal and seizure, denoted by $\mathrm{N}$ and $\mathrm{S}$, respectively. The Class N includes Set A, which contains 100 EEG signals. The Class N includes Set A, which contains 100 EEG signals without seizure acquired from five healthy volunteers with eye open while the Class S includes Set E which contains 100 EEG signals with seizure acquired from five patients.

The Freiburg dataset includes 24 h-long continuous presurgical invasive recordings of 21 patients suffering from epilepsy. The sampling rate of the recorded data is $256 \mathrm{~Hz}$. A 16-bit analog to digital converter is used to record the data over 128 channels. Out of these channels, six of them are selected based on the visual analysis of an EEG specialist. For each patient, there are at least three Ictal files such that at least one of them contains a seizure event. Among Ictal files, the files preceding the seizure event are called pre-Ictal signal files, and the ones which come immediately following the seizure segment are called post-Ictal. Ictal files have recordings of signals that are at least $50 \mathrm{~min}$ far from seizure events. Both Ictal and inter-Ictal files are stored in ASCI format and contain six channels of EEG time series.

Table 1 presents a comparison of the performance of some state-of-the-art methods in terms of best total classification accuracy (ACC) using EEG database $\{\mathrm{N}, \mathrm{S}\}$. By analyzing the ACC results in the table, we notice that the methods using time-frequency features such as the methods in Boubchir et al. [8] and Boubchir et al. [24] provide a higher ACC (up to $99.33 \%$ ) than the methods using time-domain features and/or frequency-domain features - such as the methods in Redelico et al. [20], Kannathal et al. [26], and Polat and Günecs [27]. This indicates that the time-frequency features are the relevant and discriminate features allowing to improve significantly the classification results. Moreover, the use of time-frequency image related features $[6,8,24]$ achieves the best performance than the use of time-frequency signal related-features [22]. In addition, other types of time-frequency features based on wavelet coefficients was proposed in Subasi [28], providing an ACC result (of 95\%) less than the result achieved by the time-frequency features and used in Boubchir et al. [6], Boubchir et al. [8], Boubchir

TABLE I: Performance comparison of different method using different features.

\begin{tabular}{|c|c|c|c|c|}
\hline Method & EEG representation & Feature extraction & Classification & Best ACC (\%) \\
\hline $\begin{array}{l}\text { Redelico } \\
\text { et al. (2017) [20] }\end{array}$ & Time domain & Entropies-based features & Logistic regression & 94.5 \\
\hline $\begin{array}{l}\text { Polat and } \\
\text { Günecs (2007) [27] }\end{array}$ & Frequency domain & Fourier transform-based features & Decision tree & 98.72 \\
\hline $\begin{array}{l}\text { Kannathal } \\
\text { et al. (2005) [26] }\end{array}$ & Time domain/frequency domain & $\begin{array}{l}\text { Time-domain features/ } \\
\text { Frequency-domain features }\end{array}$ & ANFIS & 92.22 \\
\hline Subasi (2007) [28] & Time-frequency domain & Wavelet-based features & ME network & 95 \\
\hline $\begin{array}{l}\text { Boubchir } \\
\text { et al. }(2014)[17,24]\end{array}$ & Time-frequency domain & Haralick descriptor-based features & SVM & 99 \\
\hline $\begin{array}{l}\text { Boubchir } \\
\text { et al. }(2015)[6]\end{array}$ & Time-frequency domain & image texture descriptor-based features & SVM & 98 \\
\hline $\begin{array}{l}\text { Boubchir } \\
\text { et al. (2015) [8] }\end{array}$ & Time-frequency domain & LBP descriptor-based features & SVM & 99.33 \\
\hline $\begin{array}{l}\text { Mohammadi } \\
\text { et al. (2017) [10] }\end{array}$ & Time-frequency domain & $\begin{array}{l}\text { Time-frequency flux, time-frequency } \\
\text { flatness, and time-frequency entropy }\end{array}$ & & 97.5 \\
\hline
\end{tabular}


et al. [22], and Boubchir et al. [24]. Finally, the method in Boubchir et al. [8] and Mohammadi et al. [10] is the most promising methods for detecting and classifying the EEG seizure with high accuracy; the time-frequency flux is the best performing time-frequency feature as it achieved the AUC of 0.94 in Mohammadi et al. [10].

\section{CONCLUSION}

A discriminant feature plays a crucial rule in the performance of an automatic epileptic seizure detection system. Feature can be extracted from the time, frequency, and joint timefrequency domains. Different features such as IF, entropy, texture, and statistical features have been proposed by different researchers. In this paper, we proposed a review of EEG features that have been proposed to characterize the epileptic seizure activities for the purpose of EEG seizure detection and classification. The analysis of these features has shown that time-frequency features, especially those based on time-frequency image description, are the most relevant and discriminate features for detecting and classifying the EEG seizure with high accuracy. Our future work will focus on adapting the EEG time-frequency features to classify the epileptic seizure activities with their degree of severity.

\section{REFERENCES}

[1] R. S. Fisher, W. V. E. Boas, W. Blume, C. Elger, P. Genton, P. Lee and J. Jr. Engel. "Epileptic seizures and epilepsy: Definitions proposed by the international league against epilepsy (ILAE) and the international bureau for epilepsy (IBE)." Epilepsia, vol. 46, no. 4, pp. 470-472, 2005.

[2] R. S. Fisher, C. Acevedo, A. Arzimanoglou, A. Bogacz, J. H. Cross, C. E. Elger, J. Engel, L. Forsgren, J. A. French, M. Glynn, D. C. Hesdorffer, B. I. Lee, G. W. Mathern, S. L. Moshé, E. Perucca, I. E. Scheffer, T. Tomson, M. Watanabe and S. Wiebe. "ILAE official report: A practical clinical definition of epilepsy." Epilepsia, vol. 55, no. 4, pp. 475-482, 2014.

[3] B. Abou-Khalil and K. E. Misulis. "Atlas of EEG and Seizure Semiology: Text with DVD." Butterworth-Heinemann, United Kingdom, 2005.

[4] H. R. Mohseni, A. Maghsoudi and M. B. Shamsollahi. Seizure detection in EEG signals: A comparison of different approaches. International Conference of the IEEE Engineering in Medicine and Biology Society, vol. 2006, pp. 6724-6727, 2006.

[5] B. Boashash, L. Boubchir and G. Azemi. "A methodology for timefrequency image processing applied to the classification of nonstationary multichannel signals using instantaneous frequency descriptors with application to newborn EEG signals." EURASIP Journal on Advances in Signal Processing, vol. 2012, no. 1, pp. 117, 2012.

[6] L. Boubchir, S. Al-Maadeed, A. Bouridane and A. A. Chérif. "Time-frequency Image Descriptors-based Features for EEG Epileptic Seizure Activities Detection and Classification." In: 2015
IEEE International Conference on Acoustics, Speech and Signal Processing (ICASSP), pp. 867-871, 2015.

[7] J. Gotman. "Automatic seizure detection: Improvements and evaluation." Electroencephalography and clinical Neurophysiology, vol. 76, no. 4, pp. 317-324, 1990.

[8] L. Boubchir, S. Al-Maadeed, A. Bouridane, and A. A. Chérif. "Classification of EEG signals for detection of epileptic seizure activities based on LBP descriptor of time-frequency images." In: 2015 IEEE International Conference on Image Processing (ICIP), 2015, pp. 3758-3762.

[9] L. Boubchir, S. Al-Maadeed and A. Bouridane. "On the use of time-frequency features for detecting and classifying epileptic seizure activities in non-stationary EEG signals." In: 2014 IEEE International Conference on Acoustics, Speech and Signal Processing (ICASSP), 2014, pp. 5889-5893.

[10] M. Mohammadi, N. A. Khan and A. A. Pouyan. "Automatic seizure detection using a highly adaptive directional time-frequency distribution." Multidimensional Systems and Signal Processing, vol. 29, no. 4, pp. 1661-1678, 2018.

[11] S. Dong, B. Boashash, G. Azemi, B. E. Lingwood and P. B. Colditz. "Automated detection of perinatal hypoxia using time-frequencybased heart rate variability features." Medical and Biological Engineering and Computing, vol. 52, no. 2, pp. 183-191, 2014.

[12] B. Boashash, N. A. Khan and T. Ben-Jabeur. "Time-frequency features for pattern recognition using high-resolution TFDs: A tutorial review." Digital Signal Processing, vol. 40, pp. 1-30, 2015.

[13] B. Boashash, G. Azemi and N. A. Khan. "Principles of timefrequency feature extraction for change detection in non-stationary signals: Applications to newborn EEG abnormality detection." Pattern Recognition, vol. 48, no. 3, pp. 616-627, 2015.

[14] S. Sameh and Z. Lachiri. "Multiclass support vector machines for environmental sounds classification in visual domain based on logGabor filters." International Journal of Speech Technology, vol. 16, no. 2, pp. 203-213, 2013.

[15] L. Boubchir and B. Boashash. "Wavelet denoising based on the MAP estimation using the BKF prior with application to images and EEG signals." IEEE Transactions on Signal Processing, vol. 61, no. 8, pp. 1880-1894, 2013.

[16] J. Löfhede, M. Thordstein, N. Löfgren, A. Flisberg, M. RosaZurera, I. Kjellmer and K. Lindecrantz. "Automatic classification of background EEG activity in healthy and sick neonates." Journal of Neural Engineering, vol. 7, no. 1, p. 16007, 2010.

[17] B. R. Greene, S. Faul, W. P. Marnane, G. Lightbody, I. Korotchikova and G. B. Boylan. "A comparison of quantitative EEG features for neonatal seizure detection." Clinical Neurophysiology, vol. 119, no. 6, pp. 1248-1261, 2008.

[18] L. Boubchir, B. Daachi and V. Pangracious. "A Review of Feature Extraction for EEG Epileptic Seizure Detection and Classification." In: Telecommunications and Signal Processing (TSP), 2017 40 th International Conference on, pp. 456-460, 2017.

[19] A. Aarabi, F. Wallois and R. Grebe. "Automated neonatal seizure detection: A multistage classification system through feature selection based on relevance and redundancy analysis." Clinical Neurophysiology, vol. 117, no. 2, pp. 328-340, 2006.

[20] F. Redelico, F. Traversaro, M. Garcia, W. Silva, O. Rosso and M. Risk. "Classification of normal and pre-ictal eeg signals using permutation entropies and a generalized linear model as a classifier." Entropy, vol. 19, no. 2, p. 72, 2017.

[21] M. Mohammadi, A. A. Pouyan, N. A. Khan and V. Abolghasemi. "Locally optimized adaptive directional time-frequency 
distributions." Circuits, Systems, and Signal Processing, vol. 37, no. 8, pp. 3154-3174, 2018.

[22] L. Boubchir, S. Al-Maadeed and A. Bouridane. "Effectiveness of combined time-frequency imageand signal-based features for improving the detection and classification of epileptic seizure activities in EEG signals." In: 2014 International Conference on Control, Decision and Information Technologies (CoDIT), 2014, pp. 673-678.

[23] R. G. Andrzejak, K. Lehnertz, F. Mormann, C. Rieke, P. David and C. E. Elger. "Indications of nonlinear deterministic and finite-dimensional structures in time series of brain electrical activity: Dependence on recording region and brain state." Physical Review E, vol. 64, no. 6, p. 61907, 2001.

[24] L. Boubchir, S. Al-Maadeed, and A. Bouridane. "Haralick feature extraction from time-frequency images for epileptic seizure detection and classification of EEG data." In: $26^{\text {th }}$ International
Conference on Microelectronics (ICM), 2014, pp. 32-35.

[25] M. Ihle, H. Feldwisch-Drentrup, C. A. Teixeira, A. Witon, B. Schelter, J. Timmer and A. Schulze-Bonhage. "EPILEPSIAE-A European epilepsy database." Computer Methods and Programs in Biomedicine, vol. 106, no. 3, pp. 127-138, 2012.

[26] N. Kannathal, M. L. Choo, U. R. Acharya and P. K. Sadasivan. "Entropies for detection of epilepsy in EEG." Computer Methods and Programs in Biomedicine, vol. 80, no. 3, pp. 187-194, 2005.

[27] K. Polat and S. Günecs. "Classification of epileptiform EEG using a hybrid system based on decision tree classifier and fast Fourier transform." Applied Mathematics and Computation, vol. 187, no. 2, pp. 1017-1026, 2007.

[28] A. Subasi. "EEG signal classification using wavelet feature extraction and a mixture of expert model." Expert Systems with Applications, vol. 32, no. 4, pp. 1084-1093, 2007. 